\title{
PARAMETER IDENTIFICATION FOR NONLINEAR ILL-POSED PROBLEMS
}

\author{
Li Li, Bo Han \\ Department of Mathematics, Harbin Institute of Technology, 150001 \\ Harbin, P.R.China, lily93480@sohu.com
}

\begin{abstract}
Since the classical iterative methods for solving nonlinear ill-posed problems are locally convergent, this paper constructs a robust and widely convergent method for identifying parameter based on homotopy algorithm, and investigates this method's convergence in the light of Lyapunov theory. Furthermore, we consider 1-D elliptic type equation to testify that the homotopy regularization can identify the parameter effectively.
\end{abstract}

Keywords- Parameter Identification, Ill-Posed, Homotopy Regularization, Homotopy Parameter

\section{INTRODUCTION}

In general, the nonlinear inverse problems for parameter identification of $q(x)$ are formulated as

$$
F(q)=0
$$

where $F(\cdot): Q \subset X \rightarrow Y$ is a nonlinear Fréchet differential operator, and $X, Y$ are Banach spaces. We may as well assume $q^{*}$ is one of the solutions of Eq. (1). Generally speaking, parameter identification aims to reconstruct the unknown function. The coefficients, the right side of the differential equations, etc, can be the parameters which will be inverted. Hence, the parameter identification is a very interesting inverse problem, whether in theory or in practice. In 2002, Dennis W.Brewer and Robert K.Powers investigated the identification of parameters in a Volterra integro-differential system with a weakly singular kernel [3]. In 2006, S.Kim and K.L.Kreider studied the parameter identification in nonlinear elastic and viscoelastic plates by solving material properties of the plate [15]. Since the identification of parameter belongs to the scope of the inverse problems, and inverse problems are usually ill-posed in Hadamard's sense, we need to seek some effective regularization methods to solve these problems. Tikhonov regularization [6,1,7] and iterative methods [9,10,12] are very useful regularization techniques for solving nonlinear ill-posed problems. For example, iteratively regularized Gauss-Newton method [2]:

$$
q_{k+1}=q_{k}-\left[F^{\prime}\left(q_{k}\right)^{*} F^{\prime}\left(q_{k}\right)+\alpha_{k} I\right]^{-1}\left[F^{\prime}\left(q_{k}\right)^{*} F\left(q_{k}\right)+\alpha_{k}\left(q_{k}-q_{0}\right)\right]
$$

Levenberg-Marquadt method [11]

$$
q_{k+1}=q_{k}-\left[F^{\prime}\left(q_{k}\right)^{*} F^{\prime}\left(q_{k}\right)+\alpha_{k} I\right]^{-1} F^{\prime}\left(q_{k}\right)^{*} F\left(q_{k}\right)
$$


and iterative multilevel algorithm [13] are attractive methods for solving nonlinear illposed problems for their straightforward implementation. Here the mark * denotes the adjoint of the operator. These iterative methods all have good convergent properties. However, these methods have limitation. They are locally convergent, that is to say, the convergence depends highly on good initial estimates. Therefore, it is necessary to look for other better methods.

It is well known that homotopy method is an important tool in nonlinear problems, which has widely convergent property. Therefore, this paper introduces Newton homotopy method, and constructs a new and widely convergent method for solving nonlinear ill-posed problem (1). Then Lyapunov theory is introduced to prove the stability of the dynamical system method. The reason that we choose the dynamical system methods is that the dynamical system methods obtain the convergence theorem under less restrictive conditions on the equation than the theorems known for the corresponding discrete methods (or so-called iterative methods). Numerical performance listed in the 4th section shows the method's effectiveness.

\section{HOMOTOPY THEORY AND DYNAMICAL SYSTEM METHOD}

Firstly, we briefly introduce the homotopy theory for solving nonlinear operator equations (1). The concept of homotopy was first formulated by Poincaré around 1900. More details can refer to Watson [8].

In short, Homotopy is a continuous transformation from one function to another. A homotopy function $H(q, \lambda)$ between these two functions $F$ and $G$ from a space $X$ to a space $Y$ is a continuous map

$$
H: X \times[0,1] \mapsto Y
$$

which is constructed by adding a scalar homotopy parameter $\lambda \in[0,1]$ and a simple function $G(q)$ to the existing function $F(q)$, such that

$$
H(q, 0)=G(q), \quad H(q, 1)=F(q)
$$

The premise is that the solution of $G(q)=0$ has been known. For example, we may choose an initial parameter $q_{0}(x)$ arbitrarily, and take $G(q)=F(q)-F\left(q_{0}\right)$. Hence, $q_{0}$ is a solution of $G(q)=0$. Then a Newton homotopy can be constructed as follows:

$$
F(q)-(1-\lambda) F\left(q_{0}\right)=0, \quad 0 \leq \lambda \leq 1
$$

We wish that there exists a curve $q=q(\lambda), \lambda \in[0,1]$, which satisfies the following homotopy equation

$$
H(q, \lambda)=0
$$

We proceed from $q(0)=q_{0}$ along this curve $q=q(\lambda)$, and then obtain a solution $q(1)=q^{*}$, which is a solution of Eq. (1). We apply a transformation $\lambda=q-e^{-a \hat{\lambda}}$ ( $a>0$ is a constant) to map $\lambda \in[0,1]$ to $\hat{\lambda} \in[0, \infty)$. For convenience, we still use $\lambda$ in the subsequent sections, but $\lambda \in[0, \infty)$. We rewrite Eq. (2) as

$$
F(q)-e^{-a \lambda} F\left(q_{0}\right)=0, \quad \lambda \in[0, \infty)
$$


Differentiating each side of Eq. (3) with respect to $\lambda$, we have

$$
F^{\prime}(q) \dot{q}(\lambda)=-a e^{-a \lambda} F\left(q_{0}\right)
$$

In order to avoid the ill-posed inversion of Fréchet differential operator, one regularizes this problem and obtains the following parameter differential regularization:

$$
\begin{aligned}
& \dot{q}(\lambda)=-a e^{-a \lambda}\left[F^{\prime}(q)^{*} F^{\prime}(q)+\alpha(\lambda) I\right]^{-1} F^{\prime}(q)^{*} F\left(q_{0}\right), \\
& q(0)=q_{0}, \quad \lambda \in[0, \infty)
\end{aligned}
$$

\section{LYAPUNOV THEORY AND CONVERGENCE THEOREM}

In this section, we firstly introduce Lyapunov stability theory for abstract nonlinear dynamical system in Banach spaces, and use this stability theory to prove the parameter differential regularization to be convergent.

Let $X$ be an infinite dimensional Banach space and $Q$ be an open or closed subset containing 0 . For a map $\Phi$ from $Q \times R^{+} \rightarrow X$, we consider a general dynamical system

$$
\begin{aligned}
& \dot{e}(\lambda)=\Phi(e(\lambda), \lambda), \quad \lambda \in[0, \infty) \\
& e(0)=e_{0}
\end{aligned}
$$

Definition 3.4[5] Let $\{0\}=D \subset Q \subset X$ be a subset and $V$ be a function $V: Q \times R^{+} \rightarrow R^{+} . V(e(\lambda), \lambda)$ is called positive definite on $Q \backslash D \times R^{+}$, if

(1) $\forall(e(\lambda), \lambda) \in D \times R^{+}: V(e(\lambda), \lambda)=0$,

(2) $\forall(e(\lambda), \lambda) \in Q \backslash D \times R^{+}: V(e(\lambda), \lambda)>0$.

The next theorem states the sufficient condition of asymptotical stability of (5).

Theorem 3.5 [4] Consider equation (6) with $\Phi(\lambda, 0)=0, e(\lambda) \in Q \subset X, \lambda \geq \lambda_{0}$. If a function $V(\lambda, e(\lambda))$ can be found, defined in a neighborhood of $e(\lambda)=0$, which for $\lambda \geq \lambda_{0}$ is positive definite in this neighborhood with negative definite orbital derivative, the solution of $e(\lambda)=0$ is asymptotically stable.

Theorem 3.6 Assume $q^{*}$ is locally unique. Let Eq. (5) holds. Moreover, we assume that

$$
\left\|F^{\prime}(q)\right\| \leq L<1, \quad q \in B_{\rho}\left(q_{0}\right)
$$

and $\alpha(\lambda)>0$ is continuously differentiable, monotonically decreases as $\lambda \rightarrow \infty$,

$$
\begin{gathered}
C_{\alpha}:=\max _{\lambda \in[0, \infty)} \frac{\alpha(0)|\dot{\alpha}(\lambda)|}{\alpha^{2}(\lambda)} \\
|\dot{\alpha}(\lambda)| \geq 2 a+C_{\alpha}
\end{gathered}
$$

Here $a$ is the constant in Eq. (3). Then, the parameter estimator $q(\lambda)$ defined according to Eq. (5) satisfies $q(\lambda) \rightarrow q^{*}$ as $\lambda \rightarrow \infty$.

Proof. Because of $e(\lambda)=q(\lambda)-q^{*}$ and Eq. (5), we investigate the following dynamical system 


$$
\begin{aligned}
& \dot{e}(\lambda)=-a e^{-a \lambda}\left[F^{\prime}(q)^{*} F^{\prime}(q)+\alpha(\lambda) I\right]^{-1} F^{\prime}(q)^{*} F\left(q_{0}\right) \\
& e(0)=q(0)-q^{*}
\end{aligned}
$$

Now we take Lyapunov function as

$$
V(e(\lambda), \lambda):=\alpha(\lambda)<e(\lambda), e(\lambda)>
$$

Because of $\alpha(\lambda)>0$, it is easy to see that $V(\lambda, e(\lambda))$ is positive define in $Q \backslash\{0\}$. Next, $V(e(\lambda), \lambda)=0$ when $e(\lambda)=0$. Furthermore,

$$
\begin{aligned}
\dot{V}_{\lambda} & :=\frac{d}{d \lambda} V \\
& =\dot{\alpha}(\lambda)<e(\lambda), e(\lambda)>+2 \alpha(\lambda)<\dot{e}(\lambda), e(\lambda)> \\
& =-\mid \dot{\alpha}(\lambda)\|e(\lambda)\|^{2}-2 \alpha(\lambda) a e^{-a \lambda}<\left[F^{\prime}(q)^{*} F^{\prime}(q)+\alpha(\lambda) I\right]^{-1} F^{\prime}(q)^{*} F\left(q_{0}\right), e(\lambda)>
\end{aligned}
$$

In the following, we mainly focus on the second item of Eq. (10).

Since $\left\|F^{\prime}(q)\right\| \leq L<1, \quad q \in B_{\rho}\left(q_{0}\right)$, and $\alpha(\lambda)$ is positive and monotonically decreases,

$$
\begin{aligned}
\left\|q(\lambda)-q_{0}\right\| & =\left\|\int_{0}^{\lambda} \dot{q}(\tau) d \tau\right\|=\left\|-a \int_{0}^{\lambda} e^{-a \tau}\left[F^{\prime}(q(\tau))^{*} F^{\prime}(q(\tau))+\alpha(\tau) I\right]^{-1} F^{\prime}(q(\tau))^{*} F\left(q_{0}\right) d \tau\right\| \\
& \leq a L\left\|F\left(q_{0}\right)\right\| \int_{0}^{\lambda} \frac{e^{-a \tau}}{\alpha(\tau)} d \tau \\
& \leq \frac{a L\left\|F\left(q_{0}\right)\right\|}{\alpha(\lambda)} \int_{0}^{\lambda} e^{-a \tau} d \tau \\
& =\frac{L\left\|F\left(q_{0}\right)\right\|}{\alpha(\lambda)}\left(1-e^{-a \lambda}\right)
\end{aligned}
$$

Since

$$
\left\|F\left(q_{0}\right)\right\|=\left\|F\left(q_{0}\right)-F\left(q^{*}\right)\right\| \leq L\left\|q_{0}-q^{*}\right\| \leq L\left[\left\|q_{0}-q(\lambda)\right\|+\left\|q(\lambda)-q^{*}\right\|\right]
$$

we get

$$
\left\|q(\lambda)-q_{0}\right\| \leq \frac{L^{2}}{\alpha(\lambda)}\left(1-e^{-a \lambda}\right)\left\|q_{0}-q(\lambda)\right\|+\frac{L^{2}}{\alpha(\lambda)}\left(1-e^{-a \lambda}\right)\|e(\lambda)\|
$$

Therefore,

$$
\left\|q(\lambda)-q_{0}\right\| \leq \frac{L^{2}\left(1-e^{-a \lambda}\right)}{\alpha(\lambda)-L^{2}\left(1-e^{-a \lambda}\right)}\|e(\lambda)\|
$$

From Eq. (12) and Eq. (13), we can get

$$
\begin{aligned}
& -2 a \alpha(\lambda) e^{-a \lambda}<\left[F^{\prime}(q(\lambda))^{*} F^{\prime}(q(\lambda))+\alpha(\lambda) I\right]^{-1} F^{\prime}(q(\lambda))^{*} F\left(q_{0}\right), e(\lambda)> \\
\leq & 2 a \alpha(\lambda) e^{-a \lambda} \frac{L}{\alpha(\lambda)}\left\|F\left(q_{0}\right)\right\|\|e(\lambda)\| \\
\leq & 2 a e^{-a \lambda} L^{2} \frac{\alpha(\lambda)}{\alpha(\lambda)-L^{2}\left(1-e^{-a \lambda}\right)}\|e(\lambda)\|^{2}
\end{aligned}
$$


Hence

$$
\begin{aligned}
\dot{V}(\lambda) & \leq-|\dot{\alpha}(\lambda)| \cdot\|e(\lambda)\|^{2}+\frac{2 a e^{-a \lambda} L^{2} \alpha(\lambda)}{\alpha(\lambda)-L^{2}\left(1-e^{-a \lambda}\right)}\|e(\lambda)\|^{2} \\
& =-\left[|\dot{\alpha}(\lambda)|-\frac{2 a e^{-a \lambda} L^{2} \alpha(\lambda)}{\alpha(\lambda)-L^{2}\left(1-e^{-a \lambda}\right)}\right]\|e(\lambda)\|^{2}
\end{aligned}
$$

From Eq. (7) and Eq. (8), $\dot{V}(\lambda)<0$.

Up to now, all of the conditions of Theorem 3.5 are satisfied. Hence $q(\lambda)$ converges to $q^{*}$ asymptotically as $\lambda \rightarrow \infty$. The proof is completed.

\section{NUMERICAL PERFORMANCE}

In this section one numerical example for the solution of the parameter estimation problem, to estimate the parameter $q$ in

$$
\begin{aligned}
& -\left(q u_{t}\right)_{t}=f, \\
& u(0)=g_{0}, \quad u(1)=g_{1}
\end{aligned}
$$

from measurements of the $u$ in $[0,1]$, is presented. This parameter estimation problem can be formulated in terms of a nonlinear operator equation

$$
F(q)=u^{0}
$$

where $F$ is the operator which maps the conductivity $q$ onto its indirect measurement $u^{0} \in L^{2}(0,1)$. In [6] it was proven that this operator is Fréchetdifferentiable with a Lipschitz-continuous derivative. Moreover, we can prove that $F$ satisfies (6). Thus the general results of Section 3 are applicable.

Implementation of the homotopy regularization requires the calculation of $F(q)$, which is carried out with a finite-element scheme with ansatz for $u$ of linear splines. Using one step Euler, the homotopy regularization is discretized as

$$
q_{k+1}=q_{k}-a e^{-a k}\left[F^{\prime}\left(q_{k}\right)^{*} F^{\prime}\left(q_{k}\right)+\alpha_{k} I\right]^{-1} F^{\prime}\left(q_{k}\right)^{*} F\left(q_{0}\right)
$$

It is assumed that the parameter to be estimated is $q^{*}=1+\frac{1}{2} \sin (2 \pi t)$. We use an initial estimate $q_{0}=q^{*}+\mu t^{2}(1-t)^{2}(0.25-t)(0.75-t)$. The exact data $u^{0}$ is assumed to be $e^{t}+(1-e) t-1$ and the right-hand side $f$ of the differential equation (15) is given by $-e^{t}\left(q^{*}+\pi \cos (2 \pi t)\right)+(e-1) \pi \cos (2 \pi t)$.

$3 \%$ noise is added to the homotopy regularization and Landweber iteration, simultaneously. We compare these methods, and get the numerical results listed in Table 1 by using the PC-MATLAB.

Table 1 The Result of Homotopy and Landweber Iteration with Noise

\begin{tabular}{|c|c|c|c|c|}
\hline & $\mu$ & $\delta$ & Error $\left\|q^{*}-q^{\delta}\right\|$ & Relative error $\left\|q^{*}-q^{\delta}\right\| /\left\|q^{*}\right\|$ \\
\hline Landweber & 10 & $3 \%$ & 11.2803 & 3.2229 \\
\hline Homotopy & 10 & $3 \%$ & 0.0614 & 0.0176 \\
\hline
\end{tabular}




\section{CONCLUDING REMARKS}

This paper took advantage of the properties of Newton homotopy, and posed robust dynamical system method for identifying parameter. The numerical example proves this method to be effective.

\section{REFERENCES}

1. A. Neubauer, Tikhonov regularization for non-linear ill-posed problems: optimal convergence rates and finite-dimensional approximation, Inverse Problems 5, 541-557, 1988.

2. A. B. Bakushinskii, The problem of the convergence of the iteratively regularized Gauss-Newton method, Comput. Math. Phys. 32, 1353-1359, 1992.

3. D. W. Brewer and R. K. Powers, Parameter estimation for a Volterra integrodifferential equation, Appl. Numer. Math. 9, 307-320, 1992.

4. F. Verhulst, Nonlinear differential equations and dynamical systems, Springer-Verlag, 1990.

5. G. Q. Xu and S. P. Yung, Lyapunov stability of abstract nonlinear dynamic system in Banach space, IMA Journal of Mathematical Control and Information 20, 105-127, 2003.

6. H. W. Engl, K. Kunisch and A. Neubauer, Convergence rates for Tikhonov regularization of nonlinear ill-posed problems, Inverse Problems 5, 523-540, 1989.

7. H. W. Engl and M. Hanke and A. Neubauer, Regularization of inverse problems, (Dordrecht: Kluwer) 1996.

8. L. T. Watson, Globally convergent homotopy methods, Appl.Math.Comput. 31, 369396, 1989.

9. M. Hanke, Accelerated Landweber iterations for the solution of ill-posed equations, Numer. Math. 60, 341-373, 1991.

10. M. Hanke, A. Neubauer and O. Scherzer, A convergence analysis of the Landweber iteration for nonlinear ill-posed problems, Numer. Math. 72, 21-37, 1995.

11. M. Hanke, A regularization Levenberg-Marquardt scheme, with application to inverse groundwater filtration problems, Inverse Problems 13, 79-95, 1997.

12. O. Scherzer, A modified Landweber iteration for solving parameter estimation problems, Appl. Math. Optim. 38, 45-68, 1998.

13. O. Scherzer, An iterative multilevel algorithm for solving nonlinear ill-posed problems, Mumer. Math. 80, 579-600, 1998.

14. R. G. Airapetyan and A. G. Ramm, Dynamical systems and discrete methods for solving nonlinear ill-posed problems, Applied Mathematics Reviews, World Sci. Pub. Co. 1, 491-536, 2000.

15. S. Kim and K. L. Kreider, Parameter identification for nonlinear elastic and viscoelastic plates, Appl. Numer. Math. 56, 1538-1554, 2006.

16. L. Cao, B.Han, W. Wang, Homotopy Perturbation Method for Nonlinear Ill-posed Operator Equations, Int. J. Nonlin. Sci. Num. 10, 1319-1322, 2009 